and show which of these characteristics would determine clients' adherence. Wilk's lambda indicated the proportion of the variability that was not explained by the variable. In the computation of all statistical data, Statistical Package for Social Sciences (SPSS), version 16 was used.

Result Significant mean differences were observed among quality of health services $(p=0.007)$, income $(p=0.030)$ and perceived social stigma $(p=0.032)$. The discriminate function revealed a significant association between groups and all predictors, accounting for $71.4 \%$ of between group variability, although closer analysis of the structure matrix revealed only three significant predictors, namely quality of health services $(0.476)$ directly influences while income $(-0.381)$ and perceived social stigma $(-0.376)$ both inversely influence adherence.

Conclusion Clients' adherence towards Public-Private Mix DOTS (PPMD) treatment is an interplay of the health care delivery system and socio-economic factors.

\section{DETERMINANTS OF CLIENTS' ADHERENCE TO PUBLIC-PRIVATE MIX DOTS (PPMD) TREATMENT}

John Carlo Lim Divina. College of Nursing Graduate Studies, Cebu Normal University, Cebu City, Philippines

10.1136/bmjopen-2015-forum2015abstracts.69

Background In the latest Global Tuberculosis Report, it is estimated that one-third of the world's population is infected with Mycobacterium tuberculosis, mostly in developing countries where $95 \%$ of the cases occur (World Health Organization, 2013). In 2012, the Philippines has recorded 93, 586 sputum positive patients which is capable of infecting 10-20 persons annually, if left untreated (National Tuberculosis Control Program Manual of Procedure, 2004) thereby, not only threatening the health of individuals but that of the entire community. Hence, this major public health concern has been set as part of the target of the 6th Millennium Development Goal which is to reduce the prevalence and mortality of Tuberculosis by half in 2015. Great strides in Tuberculosis control have been accomplished in the past decades but another challenge has beset major stakeholders in the face of non-adherence to treatment which has severe human, economic and social costs. Interrupted treatment may reduce treatment efficacy and cause drug resistance, resulting in increased morbidity and mortality and further infections (Raviglione et al., as cited by the ISTC, 2006). Hence, the researcher has embarked this study to determine the predictors of clients' adherence to PPMD treatment, the critical factor in determining treatment success.

Objectives The purpose of this paper was to determine the characteristics of the two sets of respondents' in terms of the following thirteen variables, namely, age, educational attainment, income, sputum smear status, accessibility of TB DOTS unit, presence of co-morbidity, perceived self-efficacy, quality of health services, perceived social support, perceived social stigma, motivation, side-effects and adverse reactions. Moreover, the variables which can influence adherence to PPMD treatment were sought and tested through discriminant analysis.

Methods This study utilized descriptive correlational method of deriving data from adherent and non-adherent clients with pulmonary tuberculosis registered in a publicly-intiated PPMD Unit. The researcher utilized the Anti-Tuberculosis Chemotherapy Adherence Index, which consisted of both researcher-made and standardized tool. After ensuring validity and reliability, the data was processed through discriminant analysis which determined the association of the thirteen selected predictors on adherence 